\title{
Connecting frontline providers in Africa with distant experts to improve patients' outcomes through Project ECHO: a successful experience in Cameroon
}

\author{
Joel Fokom Domgue, Houston, USA, Yaounde, Cameroon; Ellen Baker, Houston, USA; Florence Manjuh, Bamenda, \\ Cameroon; Melissa Lopez, Houston, USA; Thomas Welty, Bamenda, Cameroon; Kathleen M Schmeler, Houston, USA \\ and The Cameroon Cervical Cancer Prevention ECHO collaborative group
}

As in many lower income settings, Cameroon-a country situated at the crossroads between West and Central Africa-experiences a high burden of cervical cancer, which is amplified by the HIV epidemic. In addition to lacking resources to implement sustainable population-based screening, the paucity of skilled healthcare providers contributes to this high burden of cervical cancer.

In support of government's efforts to reduce death from cervical cancer, private initiatives help to deal with this major public health concern. The Cameroon Baptist Convention Health Services (CBCHS), a not-for-profit, faith-based healthcare organization that has provided medical services in Cameroon for over 50 years, is one of these non-governmental initiatives. In
2007, in response to the large number of cases of cervical cancer seen in its facilities, the CBCHS founded the Women's Health Program (WHP). Since its inception, this program has provided cervical cancer screening to more than 95000 women in 7 of the 10 regions of Cameroon (Figure 1). In a setting where well-trained specialist physicians are rare and poorly accessible, the WHP is staffed by nurses who have been trained to use visual methods to provide cervical cancer screening and to treat cervical cancer precursor lesions with cryotherapy, thermal coagulation, and the loop electrosurgical excision procedure. With this nurse-led approach, WHP has been able to offer affordable preventive services and establish stronger links with the population. Despite the success

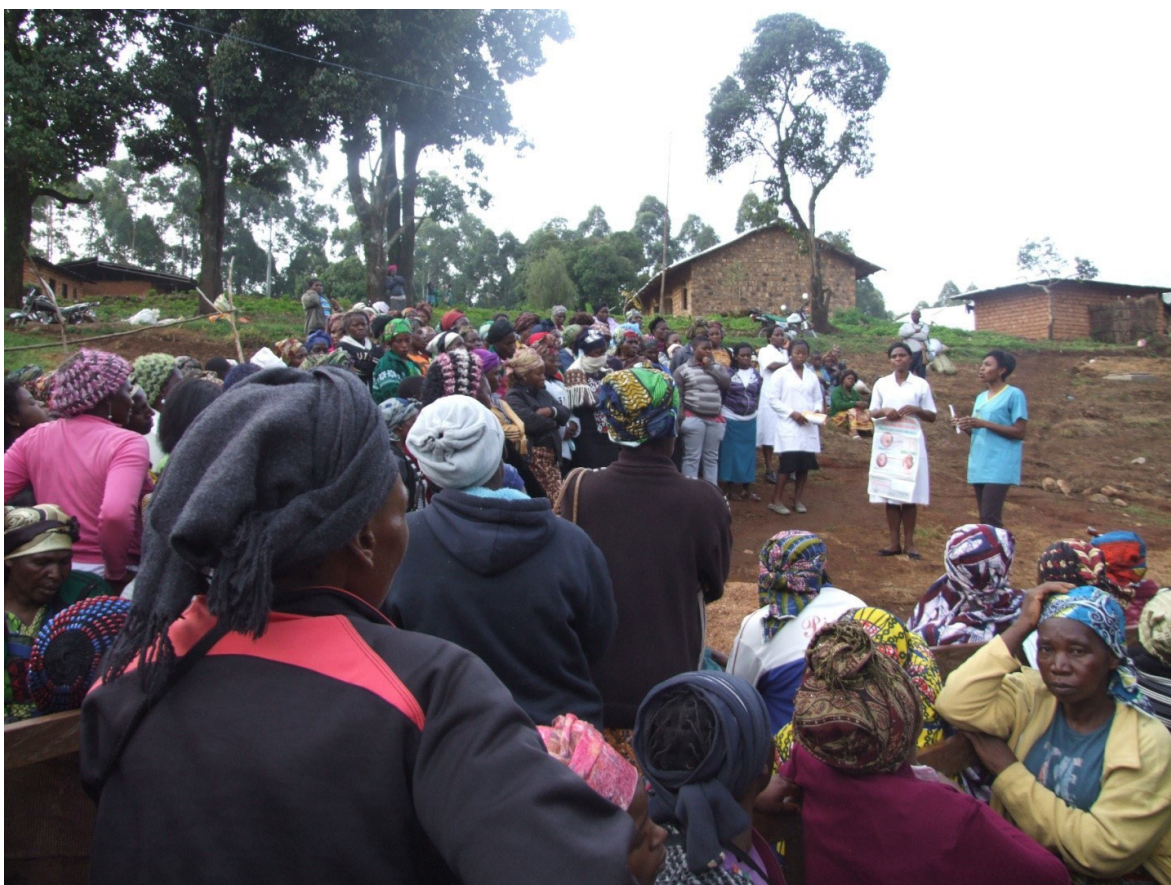

Figure 1 A campaign for cervical cancer screening organized by the Women's Health Program of the CBCHS in a rural village of Cameroon.

of this largely self-sustained program, the need for WHP providers to regularly update their skills and knowledge has emerged, as scientific discoveries are evolving rapidly. In addition, management of difficult cases has been challenging within the WHP, as most patients referred to higher level healthcare facilities could not reach these facilities for various reasons, preferring to be treated by their local caregivers.

At the beginning of my cancer prevention training at MD Anderson in November 2017, I became acquainted with a simple and inexpensive telementoring tool-the Project ECHO (extension for community healthcare outcomes) - that connects community-based providers with distant specialty consultants. This model was developed to break up the monopoly of specialized knowledge and could be helpful to the WHP to maintain standard of care services to patients at lower cost, while reducing the need for patients to travel long distances to see specialists. With the MD Anderson ECHO leadership, I introduced the ECHO model to WHP nurses who welcomed it enthusiastically. In March 2018, I traveled to Cameroon to provide local stakeholders with a better explanation of Project ECHO, what is required to set up this tool, and how it can be used for capacity building of local staff and improve patient care (Figure 2). During this trip, I assessed the expectations of WHP nurses, and received their input on how to successfully implement this telementoring model within the WHP. Then, roles and responsibilities of each stakeholder were clearly defined, and after a Project ECHO orientation session at MD Anderson a couple of months later, a formal agreement between the $\mathrm{CBCHS}$ and the $\mathrm{ECHO}$ Institute was signed. 


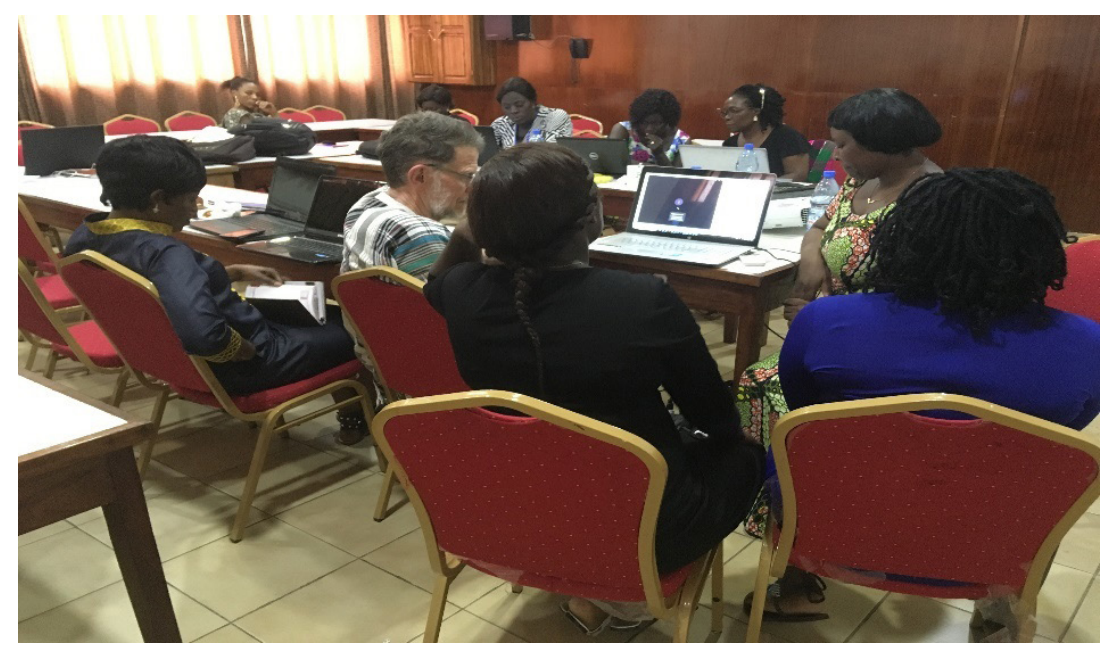

Figure 2 A meeting with Women's Health Program providers in Cameroon in March 2018, to talk about Project ECHO.

In June 2018, we officially started the Cameroon cervical cancer ECHO program. During each monthly ECHO videoconferencing session: (i) WHP nurses select and present two clinical cases to Faculty in the USA, Canada, and Africa, who provide expertise and feedback, and (ii) Faculty present a short didactic lecture on a topic of interest to WHP nurses (Figure $3 A, B$ ). These sessions are interactive, and focus on resources (including equipment and drugs) available locally that can be used to maximum advantage to provide better care to patients. Ten months later, the Cameroon ECHO program is very successful, with an increasing number of participants at each session, and positive feedback from local caregivers on the impact of this training model on their clinical practice and self-efficacy. The scope of these sessions has gradually expanded to the diagnosis and management of lower genital tract malignancies and human papillomavirus-related diseases. To date, providers from 10 other African countries have joined these ECHO sessions, and we are considering increasing the frequency of these sessions to twice a month. In view of the interest expressed by many French-speaking African countries in participating in this program, we are working with the International Gynecologic

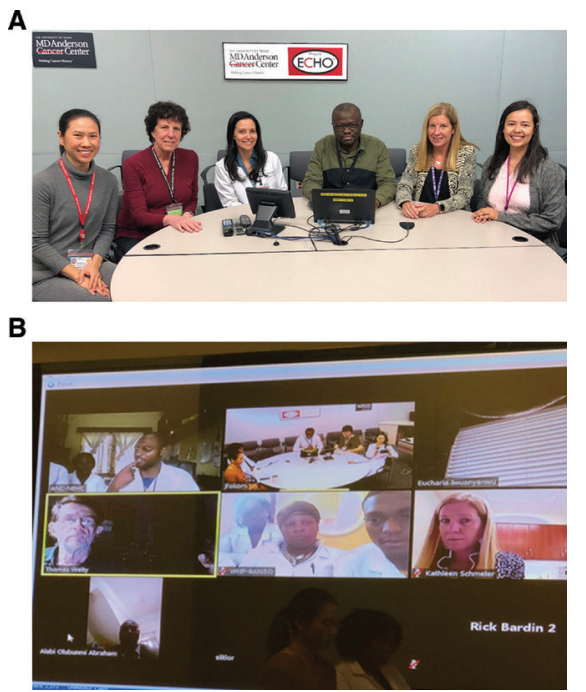

Figure 3 A Cameroon cervical cancer $\mathrm{ECHO}$ videoconference at MD Anderson3. A: Facilitors and $\mathrm{ECHO}$ staff attending in person an $\mathrm{ECHO}$ session in a dedicated conference room at MD Anderson3. B: Providers and Faculty joining an $\mathrm{ECHO}$ session from their phone or computer in Africa, the USA and Canada.
Cancer Society's (IGCS) mentorship and training committee to set up an ECHO-modeled Tumor Board in French to meet this growing need.

Correspondence to Dr Joel Fokom Domgue, Departments of Epidemiology, and Gynecologic Oncology and Reproductive Medicine, The University of Texas MD Anderson Cancer Center, Houston, TX 77030, USA; jfokom@mdanderson.org

Collaborators At MD Anderson Cancer: Sanjay Shete, Andrea Milbourne, Mila Salcedo, Natacha Phoolcharoen, Lois Ramondetta, Stella Dike, Carrie Cameron, Shine Chang, Sanjay Shete, Ernest Hawk. At the Cameroon Baptist Convention Health Services: Edith Welty, Richard Bardin, Calvin Ngalla, Divine Njodeven, Kathleen Nulah, Pius Tih. At the International Gynecologic Cancer Society: Mary Eiken, Tri Dinh. At other institutions in Canada, the USA, and Africa: Laurie Elit, Alan Waxman, David Greenspan, Michael Hicks, Groesbeck Parham, Ernest Hawk.

Contributors JFD conceived the project, identified and invited partners in Africa to participate in the project, and together with KMS and TW, invited experts in the USA, Canada, and Africa to serve as Faculty for this ECHO program. JFD wrote the first draft of the manuscript. The other contributors revised and approved the final version of the manuscript. JFD coordinates the project at MD Anderson. SS, EH, and KS supervise the project.

con Supervision

Funding This research was supported in part by the National Institutes of Health (NIH) through MD Anderson's Cancer Center Support Grant P30CA016672, and a Cancer Prevention Fellowship supported by the Cancer Prevention and Research Institute of Texas (CPRIT) grant award, RP170259 (JFD).

Competing interests None declared.

Patient consent for publication Not required.

Provenance and peer review Commissioned; internally peer reviewed.

(c) IGCS and ESGO 2019. No commercial re-use. See rights and permissions. Published by BMJ.

$$
\text { A Check for updates }
$$

To cite Fokom Domgue J, Baker E, Manjuh F, et al. Int J Gynecol Cancer 2019;29:1446-1447.

Accepted 15 April 2019

Published Online First 21 May 2019

Int J Gynecol Cancer 2019;29:1446-1447. doi:10.1136/ijgc-2019-000405 